Case Report

\title{
Temporary Denosumab Discontinuation Promotes Bone Healing of Osteonecrosis of the Jaw and Minimizes the Invasiveness of Surgery: A Case Presentation
}

\author{
Giordana Bettini ${ }^{1,2, *(\mathbb{D})}$, Giorgia Saia ${ }^{1,2}$, Federica Benetello ${ }^{3}$ and Alberto Bedogni ${ }^{1,2}$ (D) \\ 1 Unit of Maxillofacial Surgery, Department of Neurosciences, University of Padua, Via Giustiniani 2, \\ 35128 Padova, Italy; giorgia.saia@unipd.it (G.S.); alberto.bedogni@unipd.it (A.B.) \\ 2 Regional Centre for Prevention, Diagnosis and Treatment of Medication and Radiation-Related Bone Diseases \\ of the Head and Neck, Unit of Maxillofacial Surgery, Department of Neuroscience, University of Padua, \\ Via Giustiniani 2, 35128 Padua, Italy \\ 3 Dentistry Clinic, Department of Neurosciences, University of Padova, Via Giustiniani 2, 35128 Padova, Italy; \\ federica.benetello.1@studenti.unipd.it \\ * Correspondence: giordana.bettini@unipd.it; Tel.: +39-049-8212003
}

check for

updates

Citation: Bettini, G.; Saia, G.;

Benetello, F.; Bedogni, A. Temporary

Denosumab Discontinuation

Promotes Bone Healing of

Osteonecrosis of the Jaw and

Minimizes the Invasiveness of

Surgery: A Case Presentation. Oral

2022, 2, 41-48. https://doi.org/

$10.3390 /$ oral2010006

Academic Editors: Luca Testarelli

and Shankargouda Patil

Received: 30 December 2021

Accepted: 8 February 2022

Published: 16 February 2022

Publisher's Note: MDPI stays neutral with regard to jurisdictional claims in published maps and institutional affiliations.

Copyright: (c) 2022 by the authors. Licensee MDPI, Basel, Switzerland. This article is an open access article distributed under the terms and conditions of the Creative Commons Attribution (CC BY) license (https:// creativecommons.org/licenses/by/ $4.0 /)$.

\begin{abstract}
Denosumab has proved effective at low doses in increasing bone mineral density in osteoporosis patients. In contrast to high-doses antiresorptive therapy, denosumab has a transient effect on the inhibition of the bone remodeling process, suggesting that denosumab-related osteonecrosis is a self-limiting disease, with a high curative potential of surgery when performed after a proper duration of receptor activator of nuclear factor-kB ligand (RANKL) inhibitor suspension. We report the long-term clinical and radiological (Computed Tomography-CT scan) data of a patient affected by secondary osteoporosis (cancer treatment-induced bone loss-CTIBL for metastatic breast cancer) who underwent surgical treatment for stage II denosumab-related osteonecrosis of the upper maxilla 7 months after denosumab suspension. A minimally invasive approach was performed with the extraction of the first right upper molar and debridement of the surrounding alveolar bone. After surgery, the patient was followed up at three-month intervals up to 1 year, and clinical and radiological data (CT scan) were recorded at each follow-up for the early detection of signs of recurrent disease. The mucosal healing remained stable in the long term, with radiological signs of bone remodeling in the post-operative site since the 6-month follow-up. The presented case strengthens the hypothesis that denosumab induces temporary alterations of bone turnover with a predictable curative effect of minimal surgical procedures in cases of denosumab-related osteonecrosis of the jaw.
\end{abstract}

Keywords: medication-related osteonecrosis of the jaw; ONJ; denosumab; osteoporosis; treatment; surgery

\section{Introduction}

Medication-related osteonecrosis of the jaw (MRONJ) occurs more frequently in patients receiving high doses of bisphosphonates or denosumab (RANKL-inhibitor) in a monotherapy schedule [1].

At low doses (60 mg every six months), denosumab (DNB) has proved effective in increasing the bone mineral density in osteoporosis patients. Despite the highest incidence of MRONJ still being associated to bisphosphonates (BP), recent observations on low-dose DNB-induced ONJ are emerging in osteoporotic patients [2,3].

Although the pathogenesis of DNB-induced ONJ is not fully clarified, the inhibition of osteoclastic activity on the jawbone resulting in the over suppression of bone turnover is the main substantial hypothesis [4].

In contrast to bisphosphonates, DNB acts primarily as a circulating protein without sustained binding to bone surfaces, resulting in a transient effect on the inhibition of the bone remodeling process [5]. 
It has been observed that bone remodeling dramatically recovers after a proper amount of time (seven to eight months) of RANKL inhibitor suspension [6,7], reducing the effect of the DNB cumulative dosage on the risk of ONJ development in the presence of oral triggers (dental/periodontal infection or oral surgery) [8].

Accordingly, it has been suggested that DNB-related ONJ is a time-dependent process with potential self-limitation after drug suspension [7].

In this scenario, the curative potential of surgery in patients affected by DNB-related ONJ is likely to be increased as compared with bisphosphonate-related ONJ, when performed after the proper duration for the circulating dose decline.

Currently, standard medical care for MRONJ is still lacking, along with evidence about the most effective treatment strategies $[9,10]$.

Stage-dependent strategies to treat MRONJ have been originally proposed based on a clinical classification system and can be divided into medical and surgical treatments [11].

The suggested aim of treatment for mild to moderate disease (stages 1 and 2, respectively) is the control of local symptoms and infection. Surgical treatment (bone resection) is mainly reserved for advanced (stage 3) and refractory disease.

The medical treatments that are effective in the control of signs and symptoms of MRONJ are systemic antibiotics, the use of antibacterial mouth rinses (e.g., chlorhexidine) and the maintenance of good oral hygiene. Additionally, superficial debridement of exposed bony spicula can improve patient discomfort, especially at an early disease stage [10-12].

However, a high recurrence rate with impaired mucosal healing has been observed following medical treatment, resulting in prolonged therapies with poor patients' quality of life $[12,13]$.

Over the recent years, the role of surgery has been increasingly emphasized as an effective strategy for long-term healing and disease control [14,15].

Nonetheless, disease-stage strategies have been progressively modified with the introduction of a clinico-radiological staging system where both medical and surgical treatments are provided for each MRONJ stage on the basis of the real disease extent at computed tomography [16-18].

However, the strongest data are mainly provided for bisphosphonate-induced ONJ, with, at present, little evidence for denosumab-related ONJ.

We report the 1-year clinical and radiological follow-up of a breast cancer patient with cancer treatment-induced bone loss (CTIBL), who had been surgically treated for DNB-related osteonecrosis of the upper maxilla seven months after the last dose of DNB.

\section{Case Presentation}

A 54 year old women was referred to our Regional Centre at the Unit of Maxillofacial Surgery in Padua in February 2020 with spontaneous bone sequestration three months after a periodontal infection of the upper right first molar.

Her medical history was significant for non-metastatic hormone receptor-positive breast cancer diagnosed in 2018. She was initially treated with surgery and local radiotherapy, followed by risk-reducing bilateral salpingo-oophorectomy and long-term adjuvant therapy with aromatase inhibitors.

In September 2019, she completed a 2-year schedule of denosumab (60 mg s.c., every six months) for the prevention of cancer treatment induced bone loss (CTIBL). She received a total of four DNB injections with a significant improvement of the bone mineral density. She had no history of bisphosphonate therapy or radiation therapy of the head and neck.

In December 2019, she complained of moderate pain in the posterior region of her right upper maxilla. She was initially treated with antibiotic therapy (oral amoxicillin $1 \mathrm{~g}$. twice/day) without pain relief and scheduled for root canal treatment of the first upper right molar. In the meanwhile, spontaneous bone sequestration occurred at that site a few days later. The bone sequester was sent for a histological examination, which confirmed the 
presence of bone necrosis. Root canal treatment was consequently waived, and the patient was referred to our center.

At the first consultation at the outpatient clinic of the Regional Centre for Prevention, Diagnosis and Treatment of Medication and Radiation-related Bone Diseases of the Head and Neck, she presented with a painless probing bone fistula in the site of the previous bone sequestration. Mild inflammation with recession of the surrounding gingiva was observed in the absence of purulent discharge. The first upper right molar was stable without decay or fracture. No other signs were detected in the oral cavity (Figure 1).

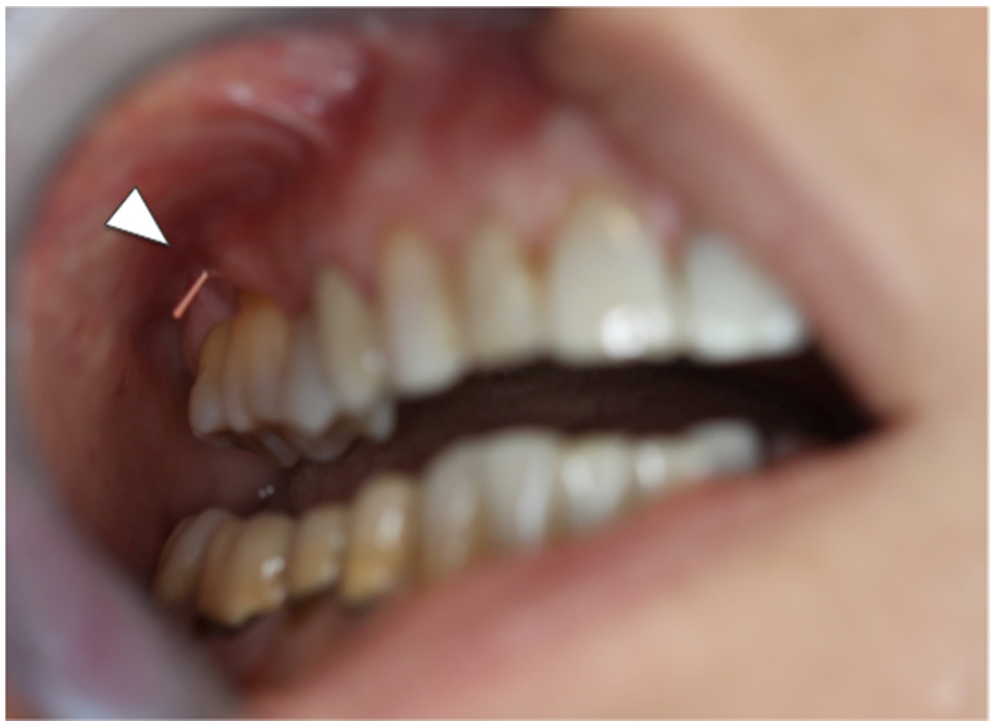

Figure 1. Intraoral patient's image at presentation showing a mucosal fistula in the vestibular buccal sulcus at the upper first right molar with mild gingival inflammation. An endodontic cone was used for dentoalveolar sinus track magnification (white arrowhead).

The panoramic radiograph showed a focal sclerosis of the alveolar bone surrounding the first right molar and a thickening of the lamina dura (Figure 2).

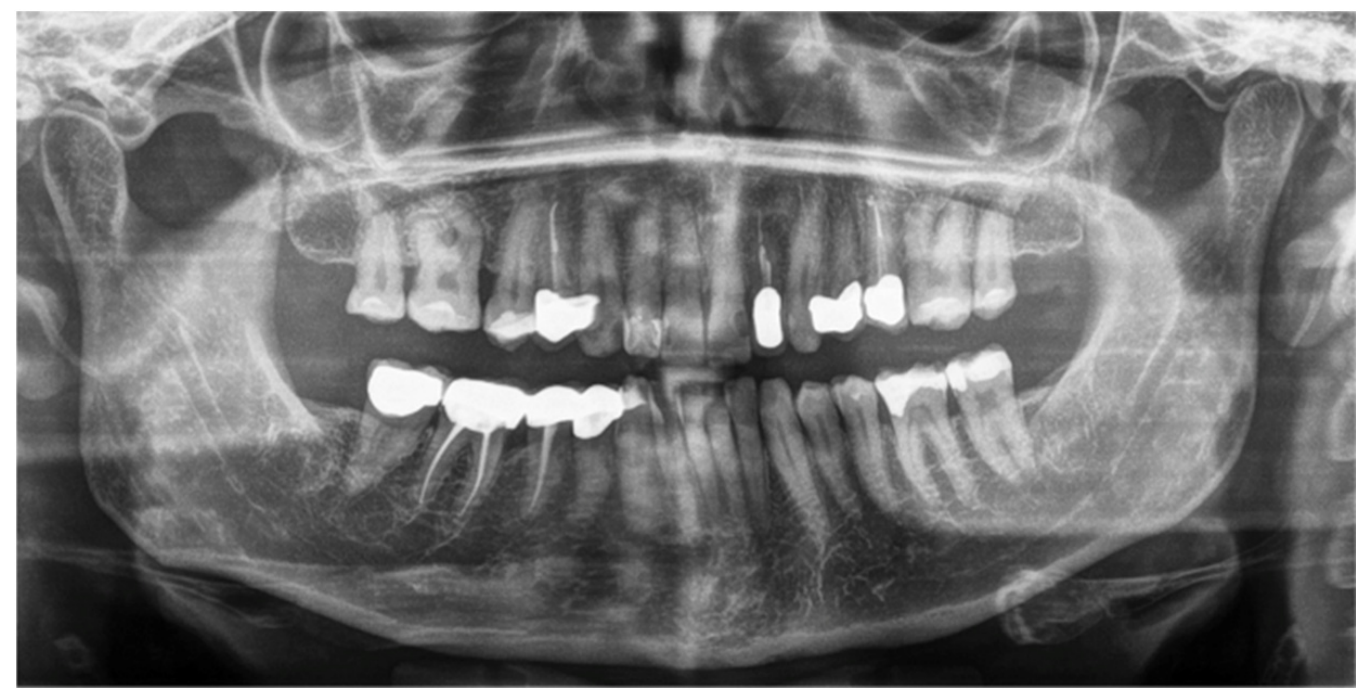

Figure 2. The panoramic radiograph was significant for aspecific signs: mild bone trabecular thickening around the first right molar roots without apical rarefaction or periodontal marginal bone reabsorption. 
At computed tomography (CT), cortical erosion and widening of the periodontal space were detected. A focal increased trabecular bone density with loss of trabecular architecture was also detected around the alveolar bone. The radiological bone changes mainly involved the area of the first right molar but also extended to the alveolar bone of the second molar. The thickening of the maxillary sinus membrane was suggestive of a process involving the entire bone height (Figure 3a,b).

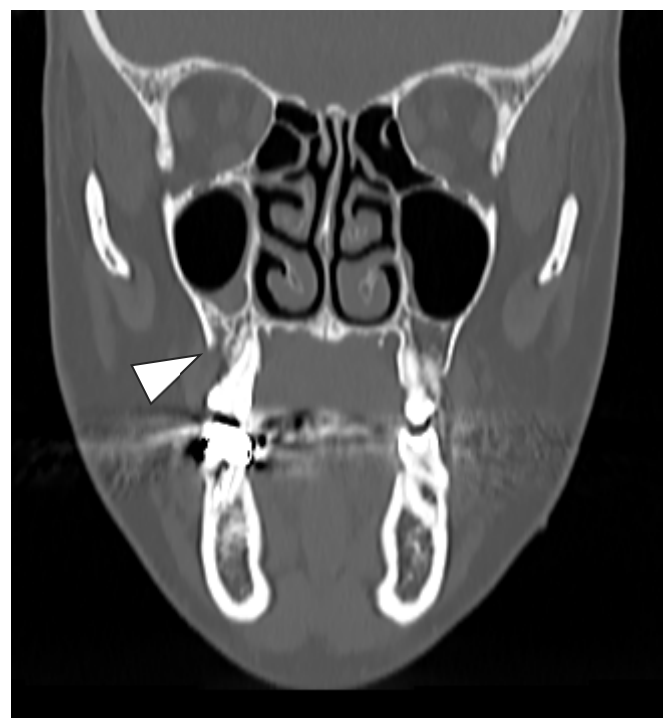

(a)

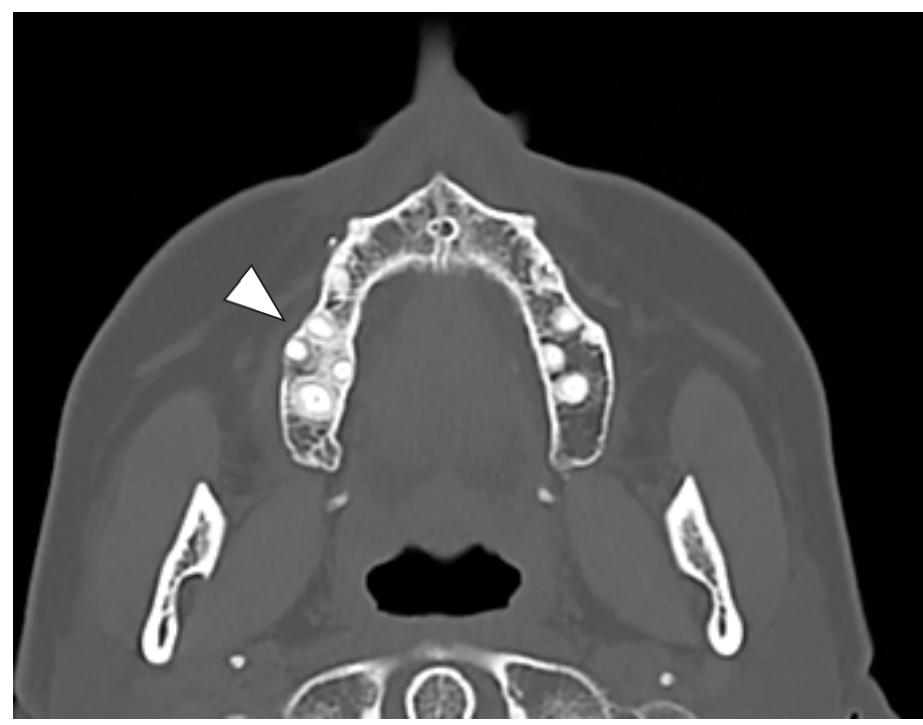

(b)

Figure 3. (a) Coronal CT view showing a cortical vestibular interruption the site of the first right molar with an irregular trabecular pattern of alveolar bone (white arrowhead); (b) Axial CT view: a focal medullar bone sclerosis entirely surrounded the first molar roots with an extension at the area of the second molar associated with a thickening of the lamina dura (white arrowhead).

The clinical and radiological signs were consistent with the diagnosis of stage IIa DNB-related ONJ according to the SICMF-SIPMO classification [17,18].

After consultation with the rheumatologist, in the presence of normal circulating biomarkers of bone mineral density, it was decided to proceed with the surgical treatment of the MRONJ site and delay DNB injection until complete mucosal healing.

On April 2021, seven months after the last dose of DNB, the patient underwent surgery. Extraction of the first right upper molar and curettage of the surrounding alveolar bone was performed. Wound closure was achieved with the elevation of a full-thickness mucoperiosteal flap. During surgery, multiple bone marrow biopsies were collected for histological examination. A wide-spectrum 7-day cycle of oral antibiotic therapy (AmoxicillinClavulanic acid $1 \mathrm{~g}$ tid, associated with Metronidazole $500 \mathrm{~m}$ tid) was administered in the post-operative course.

After surgery, the patient was followed up at three-month intervals up to one year, and clinical and radiological data (CT) were recorded at each follow-up for the early detection of clinical and radiological signs of a recurrent disease.

The post-operative course was uneventful with pain control. Mucosal healing was fully achieved within two weeks and remained stable at the 1-year follow-up.

No frank histological findings of bone necrosis were observed in the bone specimens collected during surgery. Interestingly, alveolar bone samples showed aspects of physiologic cellularity and microarchitecture (Figure 4).

The patient restarted DNB therapy 10 months after surgery when circulating biomarkers were critical for low bone mineral density (CTIBL). 
At the 6-month post-operative CT scan, new bone formation was observed in the alveolar socket of the first right upper molar, with a regular trabecular pattern. No signs of bone marrow sclerosis were detected at the right upper molar area (Figure 5a).

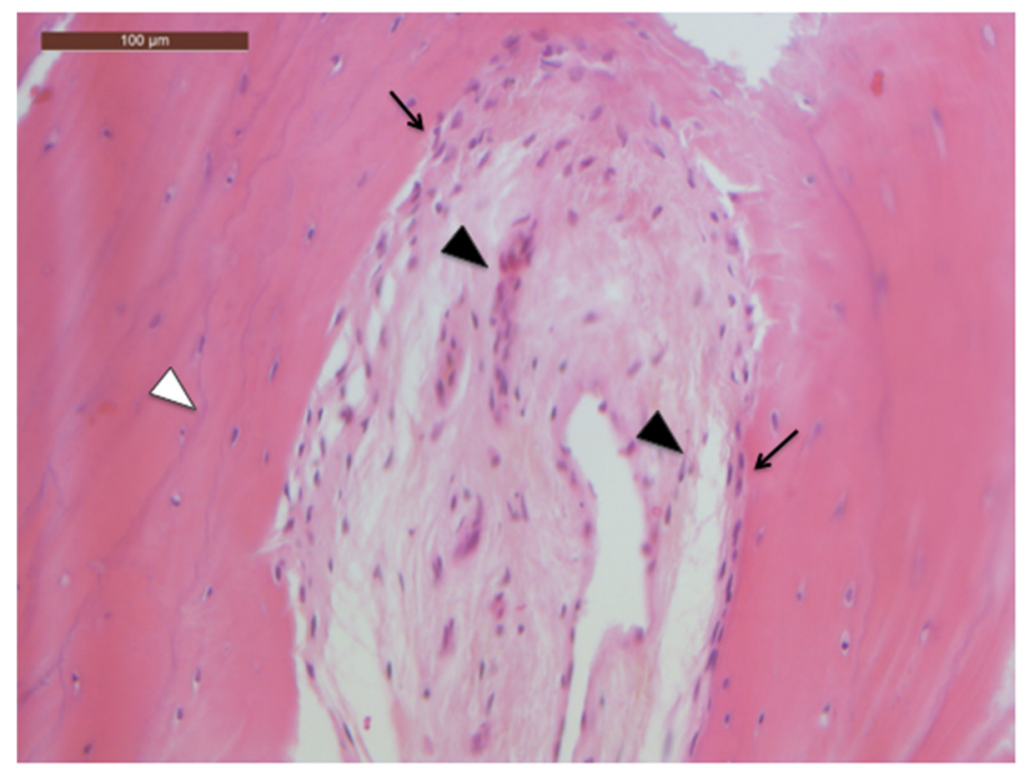

Figure 4. Histologic features of the alveolar bone specimen: normal alveolar bone pattern with lamellar bone regular microarchitecture (white arrowhead) and cellularity; the inter-trabecular space is filled with fibrous and adipose tissue infiltrating the medullar space (black arrowheads); viable osteocytes are detected at the trabecular bone surfaces (black arrows). (Hematoxylin-Eosin stain, scale bar magnification: $100 \mu \mathrm{m})$.

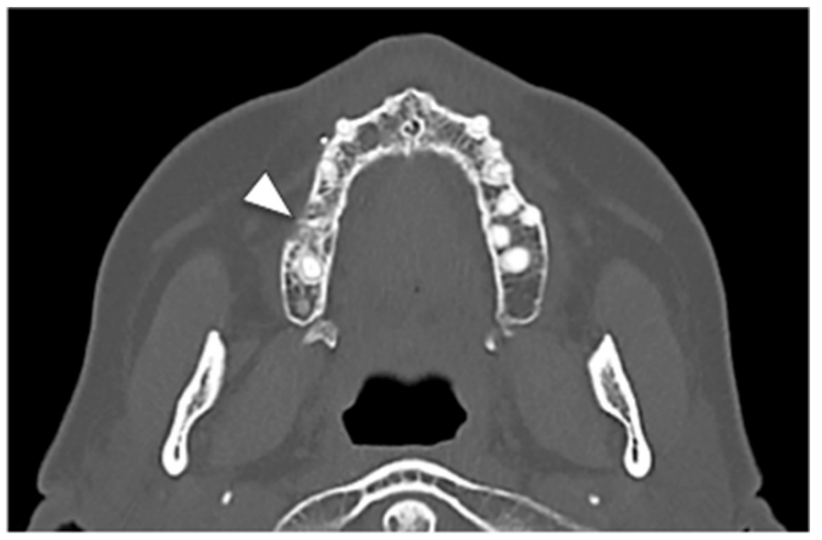

(a)

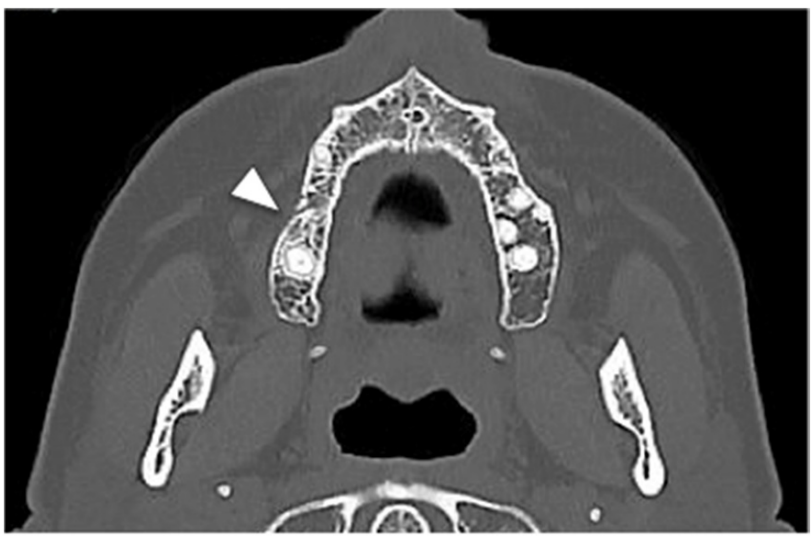

(b)

Figure 5. (a) Six-month post-operative CT axial scan with clear aspects of newly formed alveolar bone at the site of surgery (first right upper molar); a residual cortical interruption is maintained in the vestibular aspect without bone focal sclerosis (white arrowhead); (b) Axial CT at one year showing an almost complete continuity of the cortical bone with an alveolar crest width comparable to the contralateral site and resulting from bone repair (white arrowhead); the posterior region of the upper right jaw showed a regular bone architecture and trophism.

At the 1-year CT scan, no bone alterations were present (Figure 5b). 


\section{Discussion}

The presented case strengthens the hypothesis that temporary discontinuation of denosumab, when appropriate, can facilitate bone healing and reduce the invasiveness of surgery in patients with denosumab-related osteonecrosis of the jaw.

At present, the pathogenetic mechanism of DNB-related jawbone necrosis is not fully understood, and no clear evidence is available on either the efficacy of preventive drug suspension or the proper timing of this approach [12].

Recently, MRONJ treatment indications have been grouped based on the general agreement that the clinical course of disease differs between cancer and non-cancer patients receiving high- and low-dose antiresorptive treatment regimens, respectively $[8,17]$.

In general, cancer patients are addressed to extensive surgical procedures owing to the more severe evolution of the disease when compared with osteoporotic patients, irrespective of the disease stage $[8,17,19]$.

In patients affected by DNB-related ONJ under therapy for cancer induced bone fragility (CTIBL), clear indications on ONJ management strategies are still lacking.

On the one hand, the treatment indication should be tailored to these patients so as to consider them as high-risk patients as a result of the primary cancer.

For this reason, in the case presented, the bone involvement (stage II ONJ) provided the indication for the segmental resection of the diseased bone according to the treatment strategies based on the most recent MRONJ staging system [17-19].

On the other hand, the patient was administered a low-dose DNB schedule for a CTIBL condition, and this cumulative dosage has proved to have limited effects on bone tissue due to DNB pharmacokinetics [5,8].

Nevertheless, the time-dependent effect of DNB on bone suggested that DNB-related $\mathrm{ONJ}$ is a self-limiting process resulting from local reparative mechanism activation after drug cessation [7].

Based on this assumption, it has been recently suggested that surgery required for oral conditions might reasonably be performed in a protective time frame (five to seven months) between the last and subsequent DNB dose [8].

Moreover, in DNB-related ONJ, the reactivation of bone remodeling after 7-8 months of drug suspension likely limits the extension of the bone pathological involvement.

Consequently, in these cases, a less invasive surgical procedure, when performed during the protective interval of drug cessation, has the potential to be curative, resulting in a reduction of the burden of surgery in these patients.

Secondly, favoring management strategies with curative purpose in DNB-ONJ patients provided the oncologist with the possibility to ultimately continue the therapy for the control of a cancer-related condition and to improve the patient's quality of life.

\section{Conclusions}

Within its limitations, the presented case focuses attention on the need for a careful evaluation of patients as well as drug-related factors in the choice of denosumab-related ONJ treatment, balancing together with the drug prescribers the best risk/benefit ratio of different treatment sequences. Well-designed cohort studies are certainly awaited to strengthen the little evidence available so far.

Author Contributions: Conceptualization, G.B. and A.B.; methodology, A.B. and G.S., investigation, G.S. and F.B.; resources, G.B. and G.S.; data curation, G.S. and F.B.; writing-original draft preparation, G.B.; writing-review and editing, A.B. and G.S.; supervision, G.S. and F.B. All authors have read and agreed to the published version of the manuscript.

Funding: This research received no external funding.

Institutional Review Board Statement: The study was conducted according to the guidelines of the Declaration of Helsinki, and approved by the local Ethics Committee of Azienda Ospedale-Università of Padova, Italy (protocol code CESC n. 4920/AO/20; date of approval 24 September 2020). 
Informed Consent Statement: Informed consent was obtained from the subject involved in the study.

Data Availability Statement: The significant data are specified in the paper. The other data not presented in this study are available on request from the corresponding author. The data are not publicly available due to privacy restrictions.

Conflicts of Interest: Alberto Bedogni has received honoraria and travel expenses from AMGEN, Italy, for educational conferences in the period of 2017-2020. The other authors declare no conflict of interest.

\section{References}

1. Nicolatou-Galitis, O.; Schiødt, M.; Mendes, R.A.; Ripamonti, C.; Hope, S.; Drudge-Coates, L.; Niepel, D.; Van den Wyngaert, T. Medication-related osteonecrosis of the jaw: Definition and best practice for prevention, diagnosis, and treatment. Oral Surg. Oral Med. Oral Pathol. Oral Radiol. 2019, 127, 117-135. [CrossRef] [PubMed]

2. Bone, H.G.; Wagman, R.B.; Brandi, M.L.; Brown, J.P.; Chapurlat, R.; Cummings, S.R.; Czerwiński, E.; Fahrleitner-Pammer, A.; Kendler, D.L.; Lippuner, K.; et al. 10 years of denosumab treatment in postmenopausal women with osteoporosis: Results from the phase 3 randomised FREEDOM trial and open-label extension. Lancet Diabetes Endocrinol. 2017, 5, 513-523. [CrossRef]

3. Everts-Graber, J.; Lehmann, D.; Burkard, J.; Schaller, B.; Gahl, B.; Häuselmann, H.; Studer, U.; Ziswiler, H.R.; Reichenbach, S.; Lehmann, T. Risk of Osteonerosis of the jaw under Denosumab compared to Bisphosphonates in patients with osteoporosis. J. Bone Miner. Res. 2021, 37, 340-348. [CrossRef] [PubMed]

4. Ristow, O.; Gerngro, C.; Schwaiger, M.; Hohlweg-Majert, B.; Kehl, V.; Jansen, H.; Hahnefeld, L.; Koerdt, S.; Otto, S.; Pautke, C. Effect of antiresorptive drugs on bony turnover in the jaw: Denosumab compared with bisphosphonates. Br. J. Oral Maxillofac. Surg. 2014, 52, 308-313. [CrossRef]

5. McClung, M.R.; Wagman, R.B.; Miller, P.D.; Wang, A.; Lewiecki, E.M. Observations following discontinuation of long-term denosumab therapy. Osteoporos. Int. 2017, 28, 1723-1732. [CrossRef] [PubMed]

6. Malan, J.; Ettinger, K.; Naumann, E.; Beirne, O.R. The relationship of denosumab pharmacology and osteonecrosis of the jaws. Oral Surg. Oral Med. Oral Pathol. Oral Radiol. 2012, 114, 671-676. [CrossRef] [PubMed]

7. Baron, R.; Ferrari, S.; Russel, R.G. Denosumab and bisphosphonates: Different mechanisms of action and effects. Bone 2011, 48, 677-692. [CrossRef] [PubMed]

8. Campisi, G.; Mauceri, R.; Bertoldo, F.; Fusco, V.; Bedogni, A. A pragmatic window of opportunity to minimise the risk of MRONJ development in individuals with osteoporosis on Denosumab therapy: A hypothesis. Head Face Med. 2021, 17, 25-29. [CrossRef] [PubMed]

9. Beth-Tasdogan, N.H.; Mayer, B.; Hussein, H.; Zolk, O. Interventions for managing medication-related osteonecrosis of the jaw. Cochrane Database Syst. Rev. 2017, 10, CD012432. [CrossRef] [PubMed]

10. Lorenzo-Pouso, A.I.; Bagán, J.; Bagán, L.; Gándara-Vila, P.; Chamorro-Petronacci, C.M.; Castelo-Baz, P.; Blanco-Carrión, A.; Blanco-Fernández, M.Á.; Álvarez-Calderón, Ó.; Carballo, J.; et al. Medication-Related Osteonecrosis of the Jaw: A Critical Narrative Review. J. Clin. Med. 2021, 10, 4367. [CrossRef] [PubMed]

11. Ruggiero, S.L.; Dodson, T.B.; Fantasia, J.; Goodday, R.; Aghaloo, T.; Mehrotra, B.; O’Ryan, F. American Association of Oral and Maxillofacial Surgeons Position Paper on Medication-Related Osteonecrosis of the Jaw-2014 Update. J. Oral Maxillofac. Surg. 2014, 72, 1938-1956. [CrossRef] [PubMed]

12. Otto, S.; Pautke, C.; Van den Wyngaert, T.; Niepel, D.; Schiødt, M. Medication-related osteonecrosis of the jaw: Prevention, diagnosis and management in patients with cancer and bone metastases. Cancer Treat. Rev. 2018, 69, 177-187. [CrossRef] [PubMed]

13. Fliefel, R.; Troltzsch, M.; Kuhnisch, J.; Ehrenfeld, M.; Otto, S. Treatment strategies and outcomes of bisphosphonate-related osteonecrosis of the jaw (BRONJ) with characterization of patients: A systematic review. Int. J. Oral Maxillofac. Surg. 2015, 44, 568-585. [CrossRef]

14. Ristow, O.; Rückschloß, Y.; Müller, M.; Berger, M.; Kargus, S.; Pautke, C.; Engel, M.; Hoffmann, J.; Freudlsperger, C. Is the conservative non-surgical management of medication-related osteonecrosis of the jaw an appropriate treatment option for early stages? A long-term single-center cohort study. J. Cranio-Maxillofac. Surg. 2019, 47, 491-499. [CrossRef]

15. Schiodt, M.; Otto, S.; Fedele, S.; Bedogni, A.; Nicolatou-Galitis, O.; Guggenberger, R.; Herlofson, B.B.; Ristow, O.; Kofod, T. Workshop of European task force on medication-related osteonecrosis of the jaw-Current challenges. Oral Dis. 2019, 25, 1815-1821. [CrossRef] [PubMed]

16. Bedogni, A.; Fusco, V.; Agrillo, A.; Campisi, G. Learning from experience. Proposal of a refined definition and staging system for bisphosphonate-related osteonecrosis of the jaw (BRONJ). Oral Dis. 2012, 18, 621-623. [CrossRef] [PubMed]

17. Campisi, G.; Fedele, S.; Fusco, V.; Pizzo, G.; Di Fede, O.; Bedogni, A. Epidemiology, clinical manifestations, risk reduction and treatment strategies of jaw osteonecrosis in cancer patients exposed to antiresorptive agents. Future Oncol. 2014, 10, $257-275$. [CrossRef] [PubMed] 
18. Campisi, G.; Bedogni, A.; Fusco, V. Trattamento della osteonecrosi delle ossa mascellari farmaco-relata (ONJ). In Raccomandazioni Clinico-Terapeutiche Sull'osteonecrosi Delle Ossa Mascellari (ONJ) Farmaco-Relata e sua Prevenzione; Versione 2.0; Palermo University Press: Padova, Italy, 2020; pp. 261-337.

19. Bettini, G.; Saia, G.; Perra, E.S.; Tognin, L.; Franco, P.; Bedogni, G.; Bedogni, A. Curative Rates of Medication-Related Osteonecrosis of the Jaw Following Application of the SICMF-SIPMO Surgical Treatment Algorithm. Qeios 2021. [CrossRef] 